\title{
Impactos do Programa Bolsa Família no mercado de trabalho dos municípios brasileiros
}

\author{
Carlos Barbosa Correa Junior 1 \\ Leonardo Nelmi Trevisan 1 \\ Cristina Helena Pinto de Mello ${ }^{2}$ \\ 1 Pontifícia Universidade Católica de São Paulo / Programa de Estudos de Pós-Graduação em Administração, São Paulo / SP - \\ Brasil \\ ${ }^{2}$ Escola Superior de Propaganda e Marketing / Programa de Mestrado em Comportamento do consumidor, São Paulo / SP - \\ Brasil
}

Beneficiando cerca de 14 milhões de famílias brasileiras, o Programa Bolsa Família (PBF) é um dos mais importantes programas brasileiros de transferência condicionada de renda dos últimos anos. A investigação dos impactos do PBF, no mercado de trabalho formal dos 5.570 municípios brasileiros, no período de 2004 a 2013, constitui o principal objetivo deste artigo. Usando dados totais de cada um dos municípios da União, obtidos no Cadastro Único do Ministério do Desenvolvimento Social (MDS) e no Cadastro Central de Empresas do Instituto Brasileiro de Geografia e Estatística (IBGE), métodos de estimação dos mínimos quadrados ordinários (MQO), modelos de dados em painel e modelos dinâmicos, testam-se as hipóteses da associação do benefício do PBF com a quantidade de pessoal ocupado assalariado formal e com o total dos salários e de outras remunerações. Nos três modelos utilizados, os resultados indicam associação positiva para as duas hipóteses testadas.

Palavras-chave: Programa Bolsa Família; mercado de trabalho; distribuição de renda.

\section{Impactos del Programa Bolsa Familia en el mercado de trabajo de los municipios brasileños}

Al beneficiar a cerca de 14 millones de familias brasileñas, el Programa Bolsa Familia (PBF) es uno de los más importantes programas brasileños de transferencia monetaria condicionada de los últimos años. La investigación de los impactos del PBF, en el mercado de trabajo formal de los 5.570 municipios brasileños, en el período de 2004 a 2013, constituye el principal objetivo de este artículo. Con datos totales de cada uno de los municipios de la República, obtenidos del Registro Único del Ministerio de Desarrollo Social (MDS) y del Registro Central de Empresas del Instituto Brasileño de Geografía y Estadística (IBGE), métodos de estimación de los mínimos cuadrados ordinarios (MCO), modelos de datos en panel y modelos dinámicos de Arellano y Bond (1991), se prueban las hipótesis de la asociación del beneficio del PBF con la cantidad de personal ocupado asalariado formal y con el total de los salarios y de otras remuneraciones. En los tres modelos utilizados, los resultados indican una asociación positiva para las dos hipótesis probadas.

Palabras clave: Programa Bolsa Familia; mercado de trabajo; distribución de ingresos.

\section{Impacts of the Bolsa Familia Program on the labor market of Brazilian municipalities}

The Bolsa Família Program (PBF) benefits 14 million Brazilian families, and is one of the most important conditional cash transfer programs in Brazil in recent years. This articles investigates the impacts of the PBF in the formal labor market of the 5,570 Brazilian municipalities, from 2004 to 2013. The research used data from all Brazilian municipalities retrieved from the Cadastro Único (database of low-income families) of the Ministry of Social Development (MDS) and the Cadastro Central de Empresas (database of corporations) of the Brazilian Institute of Geography and Statistics (IBGE). The study adopted methods to estimate the ordinary least squares (OLS), and used panel data models as well as the dynamic models of Arellano and Bond (1991) in order to test hypotheses related to the association among the benefits from PBF, formal employment, and the total of salaries and other remunerations payed. The results indicate a positive association for the two hypotheses tested, in all three models applied.

Keywords: Bolsa Família Program; job market; income distribution. 


\section{INTRODUÇÃO}

Dentre as questões pesquisadas nas Ciências Sociais, como as voltadas ao estudo do crescimento da riqueza das nações ou as dirigidas à distribuição da riqueza e ao aumento do bem-estar da sociedade, uma terceira essencial é a que aborda justamente as inter-relações entre o crescimento e a distribuição de renda. A conexão entre a expansão do produto e a equidade social desafia pesquisadores há décadas, sem um consenso sobre suas causalidades recíprocas.

Pontusson (2005) expressa a essência do debate implícito na inter-relação mencionada. De um lado, adeptos do liberalismo sinalizam o problema do aumento do gasto público e seus efeitos nocivos ao investimento privado, acusando tais políticas de produzirem distorções no mercado, reduzindo a produtividade (Easterly \& Rebelo, 1993; Okun, 1975). De outro, defensores de políticas intervencionistas apontam os efeitos benéficos desse formato de crescimento econômico, seja no aumento do mercado interno, seja no efeito multiplicador da renda em tempos de crises econômicas (Barro, 1990, 1991; Lucas, 1988; Romer, 1986).

No caso brasileiro é possível verificar tanto o expressivo crescimento econômico, por exemplo, o da década de 1970, de 129\% (segundo o Instituto Brasileiro de Geografia e Estatística - IBGE), como o significativo aumento da desigualdade ao longo do período (segundo o IBGE). Na década de 1980, as sucessivas políticas econômicas, alternando congelamentos, hiperinflação e baixo crescimento da economia, coincidem com o agravamento da desigualdade social. No final da década de 1990, políticas públicas de teor "compensatório" começam a reverter a situação de extrema desigualdade. Intensificam-se os programas de transferência de renda condicionada (cash conditional transfers), como Bolsa Escola, Bolsa Alimentação, Auxílio Gás, Cartão Alimentação, e em 2003 vem o Programa Bolsa Família (PBF), cujo objetivo primordial é unificar esses quatro programas (Soares \& Sátyro, 2009).

Estudos publicados sobre o PBF destacam seus efeitos benéficos no que se refere a redução da pobreza, aumento da frequência escolar e diminuição da desigualdade (Bussmann \& Risco, 2012; Jacinto \& Tejada, 2009). Entretanto, permanecem como questão aberta os impactos do PBF em relação ao mercado de trabalho e à eficiência econômica.

Este artigo associa o total repassado pelo PBF em cada um dos 5.570 municípios às variáveis trabalhistas locais, especificamente a quantidade de formalizados e a massa salarial recebida. Para isso, utiliza três diferentes métodos de estimação: mínimos quadrados ordinários (MQO), modelos de dados em painel e o modelo de Arellano e Bond (1991). São encontrados coeficientes significativos de impacto do programa sobre as variáveis de interesse, com base em registros administrativos do Ministério do Desenvolvimento Social e Combate à Fome (MDS) entre os anos de 2004 e 2013. Os resultados indicam a associação positiva para as 2 hipóteses testadas, reforçando trabalhos anteriores que sugerem que as transferências de renda provenientes do PBF podem causar aumento de salários em um novo equilíbrio de mercado do município.

\section{IMPACTOS DO PROGRAMA BOLSA FAMÍLIA NO MERCADO DE TRABALHO FORMAL}

Para Arretche (1995, p. 3), "a provisão de serviços sociais, cobrindo as mais variadas formas de risco da vida individual e coletiva, tornou-se um direito assegurado pelo Estado a camadas bastante expressivas da população dos países capitalistas desenvolvidos". Fleury (1994, p. 11) argumenta que "a análise da intervenção do Estado nas questões sociais, através das chamadas políticas sociais, tem como corolário a existência de uma relação de direito positivo que se estabelece entre o cidadão e o 
seu Estado". Isso sugere que a "intervenção estatal via políticas sociais, regulando e/ou propiciando condições de manutenção e reprodução de uma parcela da população, é considerada uma função intrínseca ao Estado moderno, configurando padrões de direitos sociais próprios a cada nação" (Fleury, 1994, p. 11).

Kerstenetzky (2011) argumenta que as políticas sociais nacionais mantêm, desde a década de 1930 até a de 1970, um padrão atrelado ao modelo econômico desenvolvimentista no qual os principais grupos-alvo das políticas sociais são os trabalhadores urbanos e os funcionários públicos. Com a Constituição da República Federativa do Brasil (CF, 1988), entretanto, a assistência social brasileira passa a se inserir no âmbito da seguridade social, buscando substituir um ineficiente assistencialismo nacional por uma política pública de assistência social e promoção do bem-estar, integrando, junto com as políticas de saúde e previdência, o sistema geral de proteção social.

Com a nova CF (1988), o Estado brasileiro passa a ser o principal responsável pela melhoria da qualidade de vida da população: desenvolvem-se programas de amparo à saúde, como o Sistema Único de Saúde (SUS), e programas de transferência de renda - Bolsa Escola, Bolsa Alimentação, Auxílio Gás e Cartão Alimentação -, com o intuito de combater a pobreza e reduzir as desigualdades sociais. Em 2003, esses programas de transferência de renda se agrupam em um sistema único, entendido como mais eficiente: o PBF. Para Draibe (2009, p. 105), "entre os principais fatores apontados pelos analistas, destacam-se a simplicidade de desenho, a flexibilidade da implementação, a agilidade operacional, os relativamente baixos custos administrativos".

Consolidam-se os estudos que pesquisam os impactos do PBF no mercado de trabalho e no produto agregado (Barbosa \& Corseuil, 2014; T. Cavalcanti \& Corrêa, 2010; Foguel \& Barros, 2010; Ribas \& Soares, 2011; Tavares, 2010), com a proposição de novas teorias (D. M. Cavalcanti, Costa, Silva, \& Sampaio, 2016; Denes, Komatsu, \& Menezes, 2016; Nunes \& Mariano, 2015; Rands Barros, \& Athias, 2013). Da mesma forma, crescem os estudos das relações do benefício do PBF com o mercado de trabalho que, de maneira geral, baseiam-se na análise de dados da Pesquisa Nacional por Amostra de Domicílios (PNAD) ou da Pesquisa de Orçamentos Familiares (POF).

Para Foguel e Barros (2010), os programas de transferência condicionada de renda no Brasil não mudam o número médio de horas trabalhadas pelos homens, mas aumentam sua taxa de participação e levam a queda nas horas trabalhadas pelas mulheres, sem exercer influência sobre sua taxa de participação. Os autores estimam os efeitos dos programas condicionais de transferência de renda sobre a oferta de trabalho de mulheres e homens adultos, utilizando o painel de municípios continuamente cobertos pela PNAD ou pelo Censo do IBGE no período entre 2001 e 2005. Para Oliveira e Soares (2012), com exceção de grupos demográficos específicos, como mulheres com filhos, o desincentivo ao trabalho é muito pequeno ou inexistente. Brito e Kerstenetzky (2010) apuram os efeitos do PBF sobre o mercado de trabalho do beneficiário utilizando dados do Cadastro Único de 2008 e da PNAD de 2006. Santos, Leichsenring, Menezes, e Mendes-da-Silva (2017) analisam o efeito do PBF sobre o emprego formal da parcela pobre da população usando dados entre 2008 e 2011 do Cadastro Único combinados com dados da Relação Anual de Informações Sociais, concluindo que as chances dos beneficiários do PBF permanecerem no emprego são maiores do que para os não beneficiários.

Quanto à questão da informalidade, há trabalhos cujos resultados sugerem que o PBF aumenta a probabilidade do efeito substituição da ocupação de trabalhos formais para informais dos seus beneficiários (Marinho \& Mendes, 2013) e os que indicam que o programa não tem impacto sobre a escolha ocupacional dos beneficiários entre os postos formais e informais (Barbosa \& Corseuil, 2014). 
Pesquisas internacionais também investigam as relações entre políticas compensatórias e o mercado de trabalho (Asfaw, Davis, Dewbre, Handa, \& Winters, 2014; Kabber \& Waddington, 2015; Skoufia \& Di Maro, 2008). Alguns estudos buscam processos comparativos na realidade sul-americana aproximando as principais características dos programas de transferência condicionada e seus impactos nos mercados de trabalho regionais (Alzúa, Cruces, \& Ripani, 2012; Economic Commission for Latin America and the Caribbean \& International Labour Organization, 2015).

Entretanto, não obstante o teor das pesquisas internacionais, como também das nacionais, ainda permanecem como questão em discussão os impactos do PBF em relação ao mercado de trabalho formal.

\section{MÉTODO E DADOS}

Segundo Jacinto e Tejada (2009), os modelos sobre eficiência e igualdade englobam três linhas características de investigação: a) modelos dualistas e a hipótese do U invertido; b) curva de Kuznets e os modelos de crescimento; e c) curva de Kuznets e a escolha social. Este estudo pertence ao terceiro grupo, que incorpora as teorias do crescimento endógeno envolvendo elementos políticos de escolha social. Assim, para atingir o objetivo principal deste trabalho, 2 hipóteses são testadas:

- Hipótese H1: O benefício do PBF está associado positivamente com a quantidade de pessoal ocupado assalariado do município.

- Hipótese H2: O benefício do PBF está associado positivamente com o total dos salários e outras remunerações no município.

A pesquisa percorreu o modelo dedutivo econométrico. A primeira parte da análise estatística traz a formulação do modelo linear de variáveis de primeira diferença, ano a ano, com estimadores de MQO. Esse modelo de regressão é linear nos parâmetros e pode ser representado pela função (1) ${ }^{1}$ :

$$
\Delta \mathrm{Y}_{\mathrm{i}}=\beta_{1}+\beta_{2} \Delta \mathrm{X}_{\mathrm{i}}+\mathrm{u}_{\mathrm{i}}
$$

A segunda e a terceira partes da análise estatística trazem modelos de dados em painel. O segundo modelo pode ser representado pela função $(2)^{2}$, onde a variável dependente é explicada por um conjunto de variáveis explicativas e de um termo de erro.

$$
\mathrm{Y}_{\mathrm{i}, \mathrm{t}}=\alpha_{0}+\beta_{1} \mathrm{X}_{1 \mathrm{i}, \mathrm{t}}+\beta_{\mathrm{n}} \mathrm{X}_{\mathrm{ni,t}}+\mathrm{u}_{\mathrm{i}, \mathrm{t}}
$$

Finalmente, observando-se que, na análise de regressão que envolve séries temporais, os modelos também podem incluir valores defasados, o terceiro modelo contempla uma análise econométrica dinâmica com dados defasados autorregressivos, com valores defasados da variável dependente,

\footnotetext{
${ }^{1}$ Onde: $\Delta \mathbf{Y}_{\mathbf{i}}$ representa as diferenças da variável dependente, $\mathbf{i}$ representa a i-ésima unidade de corte transversal, $\boldsymbol{\beta}_{1}$ e $\boldsymbol{\beta}_{2}$ representam os parâmetros a ser estimados, $\Delta \mathbf{X}_{\mathrm{i}}$ representa as diferenças da variável independente, e $\mathbf{u}_{\mathrm{i}}$ representa o termo de erro independente.

${ }^{2}$ Onde: $\mathbf{Y}_{\text {it }}$ representa a i-ésima unidade de corte transversal no t-ésimo período de tempo, $\boldsymbol{\alpha}_{\mathbf{0}}$ e $\boldsymbol{\beta}_{\mathbf{1}}, \ldots, \boldsymbol{\beta}_{\mathbf{n}}$ representam os parâmetros a serem estimados, $\mathbf{X}_{1, \mathrm{it}}$ representa a primeira variável exógena, referente à i-ésima unidade de corte transversal no t-ésimo período de tempo, $\mathbf{X}_{\mathrm{n}, \mathrm{it}}$ representa a n-ésima variável exógena, referente à i-ésima unidade de corte transversal no t-ésimo período de tempo e $\mathbf{u}_{\mathrm{it}}$ representa o i-ésimo termo de erro no t-ésimo período de tempo.
} 
conforme a função $(3)^{3}$, onde a variável dependente é explicada por um conjunto de variáveis explicativas e um termo de erro.

$$
\mathrm{Y}_{\mathrm{it}}=\alpha_{0}+\beta_{1} \mathrm{X}_{1, \mathrm{it}}+\ldots+\beta_{\mathrm{n}} \mathrm{X}_{\mathrm{n}, \mathrm{it}}+\phi_{1} \mathrm{Y}_{\mathrm{it}-1}+\mathrm{u}_{\mathrm{i}, \mathrm{t}}
$$

Esse modelo autorregressivo se baseia na ideia de que a variável dependente não se ajusta imediatamente devido a alterações das variáveis explicativas e que valores passados tendem a influenciar seus próprios valores futuros. Neste estudo são utilizados os estimadores do modelo de momentos generalizados (MMG), desenvolvido em Arellano e Bond (1991), que permite obter estimadores confiáveis em modelos de dados em painel defasados.

Como variáveis, para cada um dos $5.570(\mathrm{~N})$ municípios utiliza $(\mathrm{m})$-se: a) valores totais recebidos de benefícios do PBF por todas as famílias com residência no município; b) número total de famílias beneficiadas pelo PBF; c) número total de pessoas ocupadas assalariadas e com carteira assinada com residência no município; d) valor total de salários e outras remunerações das pessoas ocupadas assalariadas e com carteira assinada com residência no município; e) valor total das unidades empresariais das pessoas ocupadas assalariadas e com carteira assinada com residência no município; e f) valor total anual do produto interno bruto (PIB) do município, conforme o Quadro 1.

\section{QUADRO 1 VARIÁVEIS DE ESTUDO}

\begin{tabular}{ll} 
Variáveis por município & \\
\hline Benefício total do PBF & Cadastro Único (MDS) \\
Número de famílias beneficiadas pelo PBF & Cadastro Único (MDS) \\
PIB municipal anual & Cadastro Central de Empresas (IBGE) \\
Salários e outras remunerações & Cadastro Central de Empresas (IBGE) \\
Pessoal ocupado assalariado no último dia do ano & Cadastro Central de Empresas (IBGE) \\
Unidades locais & Cadastro Central de Empresas (IBGE)
\end{tabular}

Fonte: Elaborado pelos autores.

Os dados são coletados por meio dos sites governamentais oficiais, assim, são considerados provenientes de fontes primárias do(a): a) IBGE, Diretoria de Pesquisas, Coordenação de Metodologia das Estatísticas de Empresas, Cadastros e Classificações, Cadastro Central de Empresas; e b) Cadastro Único, Matriz de Informação Social do Ministérrio do Desenvolvimento Social (MDS), ambos referentes aos anos de 2004 a 2013, com valores anuais ou mensais acumulados anualmente.

\footnotetext{
${ }^{3}$ Onde: $\mathbf{Y}_{\mathrm{it}} ; \mathbf{X}_{1, \mathrm{it}} ; \ldots ; \mathbf{X}_{\mathrm{n}, \mathrm{it}}$ e $\mathbf{u}_{\mathrm{it}}$ possuem as mesmas interpretações da função (4). Além disso, $\boldsymbol{\alpha}_{0} ; \boldsymbol{\beta}_{1} ; \ldots ; \boldsymbol{\beta}_{\mathrm{n}}$ continuam representando os parâmetros a ser estimados. A diferença nessa especificação é que aparece um novo parâmetro $\varphi 1$ que representa o parâmetro autorregressivo que precisará ser estimado e relaciona-se à primeira defasagem da variável $\mathbf{Y}_{\mathrm{it}}$, a saber, $\mathbf{Y}_{\mathrm{it}-1}$.
} 
A escolha do recorte do intervalo da observação empírica entre os anos de 2004 e 2013 se deve a 3 razões: a) nos anos de 2002 e de 2003, o PBF estava ou em fase de implantação ou em fase de início de funcionamento e entende-se que nessa fase poderiam ocorrer alterações de comportamento das famílias revertidas no próprio curto prazo; b) iniciando-se em 2004, já estão refletidos os efeitos perenes do programa; e c) o término da coleta em 2013 se deve à limitação de disponibilidade de dados sobre pessoal formal empregado e salários na data da realização da pesquisa.

A escolha do número de pessoas ocupadas e o salário e as remunerações médias terem como base apenas o trabalho formal, ou seja, das pessoas que trabalham com carteira assinada, tem o objetivo de analisar a evolução não apenas do emprego e dos salários, mas, também, da formalização da economia brasileira.

A escolha da utilização dos dados específicos totais de cada um dos 5.570 municípios em detrimento dos dados da PNAD ou do Censo do IBGE, que têm como unidade o indivíduo ou as famílias, justifica-se pela sugestão de "de buscar uma nova base de dados para verificar conclusões de estudos precedentes” (King, Keohane, \& Verba, 1996, p. 27). Também porque o objetivo da pesquisa não versa sobre o comportamento individual ou familiar decorrente do benefício do programa, mas, alternativamente, avalia o impacto agregado do programa. Ao usar os dados específicos totais de cada um dos municípios da União ao invés de ter como base os dados da PNAD ou do Censo do IBGE, ou seja, por não versar sobre o comportamento individual ou familiar decorrente do recebimento do programa, mas, alternativamente, por avaliar o impacto agregado das políticas nos municípios, esta pesquisa possibilita a confrontação com conclusões de estudos anteriores, desenvolvidos mediante o uso de bases de dados alternativas. Os dados são coletados diretamente de órgãos governamentais oficiais. A Tabela 1 resume os valores correntes totais das variáveis utilizadas.

\section{TABELA 1 VALORES CORRENTES ANUAIS TOTAIS DAS VARIÁVEIS PESQUISADAS}

\begin{tabular}{|c|c|c|c|c|c|c|}
\hline Ano & $\begin{array}{l}\text { Benefício do } \\
\text { PBF, em R\$ } \\
\text { milhões }\end{array}$ & $\begin{array}{c}\text { Número de famílias } \\
\text { beneficiadas pelo PBF, em } \\
\text { mil famílias }\end{array}$ & $\begin{array}{l}\text { PIB, em RS } \\
\text { bilhões }\end{array}$ & $\begin{array}{l}\text { Salários e outras } \\
\text { remunerações, em } \\
\text { R\$ bilhões }\end{array}$ & $\begin{array}{l}\text { Pessoal ocupado } \\
\text { assalariado, em mil } \\
\text { pessoas }\end{array}$ & $\begin{array}{l}\text { Unidades } \\
\text { locais, em } \\
\text { mil locais }\end{array}$ \\
\hline 2004 & 3.792 & 6.572 & 1.941 & 398 & 31.138 & 5.929 \\
\hline 2005 & 5.692 & 8.700 & 2.147 & 453 & 33.092 & 6.248 \\
\hline 2006 & 7.525 & 10.966 & 2.369 & 547 & 34.998 & 4.764 \\
\hline 2007 & 8.965 & 11.043 & 2.691 & 616 & 37.641 & 4.904 \\
\hline 2008 & 10.607 & 10.558 & 3.187 & 716 & 39.456 & 5.120 \\
\hline 2009 & 12.455 & 12.371 & 3.239 & 800 & 41.348 & 5.393 \\
\hline 2010 & 14.373 & 12.778 & 3.990 & 929 & 44.141 & 5.704 \\
\hline 2011 & 17.358 & 12.851 & 4.146 & 1.065 & 46.399 & 5.723 \\
\hline 2012 & 21.157 & 13.902 & 4.390 & 1.211 & 47.510 & 5.814 \\
\hline 2013 & 24.890 & 14.086 & 5.316 & 1.320 & 47.665 & 5.846 \\
\hline
\end{tabular}

Fonte: Elaborada pelos autores. 
A Tabela 2 traz as estatísticas descritivas referentes à variável benefício total do PBF por município entre os anos de 2004 e 2013. Observa-se que o valor médio do benefício cresceu, em média, 23,2\% ao ano (a.a.) no período.

\section{TABELA 2 ESTATÍSTICAS DESCRITIVAS DA VARIÁVEL BENEFÍCIO TOTAL DO PBF, VALORES CORRENTES, EM R\$}

\begin{tabular}{cccccc}
\hline Ano & Média & Mediana & Desvio padrão & \multicolumn{1}{c}{ Mínimo } & \multicolumn{1}{c}{ Máximo } \\
\hline 2004 & $685.427,52$ & $274.848,50$ & $1.985 .023,77$ & 168,00 & $83.629 .166,00$ \\
\hline 2005 & $1.023 .681,12$ & $443.798,50$ & $2.784 .547,98$ & 325,00 & $112.404 .678,00$ \\
\hline 2006 & $1.352 .383,42$ & $594.705,00$ & $3.574 .349,73$ & 720,00 & $129.006 .666,00$ \\
2007 & $1.611 .340,69$ & $706.125,00$ & $4.392 .482,73$ & $1.280,00$ & $151.772 .106,00$ \\
2008 & $1.906 .272,50$ & $823.372,00$ & $5.323 .984,63$ & $1.134,00$ & $172.598 .270,00$ \\
2009 & $2.238 .444,02$ & $949.381,50$ & $6.453 .458,76$ & 752,00 & $178.942 .695,00$ \\
2010 & $2.582 .695,93$ & $1.103 .867,00$ & $7.170 .577,59$ & 660,00 & $200.137 .472,00$ \\
2011 & $3.119 .206,63$ & $1.324 .975,00$ & $9.038 .200,23$ & 708,00 & $243.203 .214,00$ \\
2012 & $3.801 .751,07$ & $1.593 .996,00$ & $10.874 .276,25$ & $4.408,00$ & $317.886 .792,00$ \\
2013 & $4.469 .403,32$ & $1.878 .116,00$ & $12.995 .376,33$ & 620,00 & $430.243 .182,00$ \\
\hline
\end{tabular}

Fonte: Elaborada pelos autores.

A Tabela 3 traz as estatísticas descritivas referentes à variável número de famílias beneficiadas pelo PBF por município entre os anos de 2004 e 2013. Observa-se que o número de famílias beneficiadas pelo PBF cresceu, em média, $8,8 \%$ a.a. no período e que houve pequeno decréscimo no número de famílias beneficiadas em 2008 .

\section{TABELA 3 ESTATÍSTICAS DESCRITIVAS DA VARIÁVEL NÚMERO DE FAMÍLIAS BENEFICIADAS PELO PBF}

\begin{tabular}{|c|c|c|c|c|c|}
\hline Ano & Média & Mediana & Desvio padrão & Mínimo & Máximo \\
\hline 2004 & 1.188 & 518 & 3.666 & 2 & 165.991 \\
\hline 2005 & 1.565 & 692 & 4.253 & 1 & 167.038 \\
\hline 2006 & 1.971 & 887 & 5.689 & 3 & 224.817 \\
\hline 2007 & 1.985 & 879 & 5.742 & 2 & 204.127 \\
\hline 2008 & 1.898 & 834 & 5.649 & 1 & 201.664 \\
\hline 2009 & 2.223 & 966 & 6.607 & 2 & 177.882 \\
\hline 2010 & 2.296 & 1.006 & 6.926 & 1 & 196.699 \\
\hline 2011 & 2.309 & 1.007 & 7.009 & 1 & 195.532 \\
\hline 2012 & 2.498 & 1.073 & 7.704 & 4 & 228.078 \\
\hline 2013 & 2.529 & 1.045 & 8.584 & 3 & 347.191 \\
\hline
\end{tabular}

Fonte: Elaborada pelos autores. 
A Tabela 4 traz as estatísticas descritivas referentes à variável PIB por município entre os anos de 2004 e 2013. Observa-se que o valor médio da variável PIB cresceu, em média, 11,8\% a.a. no período.

\section{TABELA 4 ESTATISTIICAS DESCRITIVAS DOS PIBS MUNICIPAIS ANUAIS, VALORES CORRENTES, EM R\$ MIL}

\begin{tabular}{cccccc}
\hline Ano & Média & Mediana & Desvio padrão & Mínimo & Máximo \\
\hline 2004 & $349.190,35$ & $48.198,13$ & $3.723 .248,80$ & $3.183,93$ & $225.170 .381,88$ \\
\hline 2005 & $385.916,48$ & $50.904,53$ & $4.237 .693,56$ & $3.503,53$ & $261.455 .917,21$ \\
2006 & $425.859,73$ & $56.963,78$ & $4.610 .458,18$ & $3.746,16$ & $282.892 .454,76$ \\
2007 & $483.619,24$ & $64.913,05$ & $5.240 .545,80$ & $4.512,55$ & $323.154 .665,88$ \\
2008 & $572.622,07$ & $74.416,66$ & $6.173 .015,78$ & $6.492,19$ & $357.116 .681,33$ \\
2009 & $582.103,15$ & $80.588,13$ & $6.346 .376,79$ & $5.770,28$ & $389.317 .166,69$ \\
2010 & $719.171,08$ & $93.296,52$ & $8.679 .265,76$ & $7.237,63$ & $443.600 .101,65$ \\
2011 & $747.384,80$ & $103.767,21$ & $7.806 .938,17$ & $9.741,52$ & $477.005 .597,21$ \\
2012 & $791.496,87$ & $109.785,52$ & $8.181 .913,32$ & $8.342,57$ & $499.375 .400,66$ \\
2013 & $954.810,11$ & $130.191,00$ & $9.504 .351,70$ & $9.678,00$ & $570.706 .192,00$ \\
\hline
\end{tabular}

Fonte: Elaborada pelos autores.

A Tabela 5 traz as estatísticas descritivas referentes à variável salários e outras remunerações recebidas pelo pessoal ocupado assalariado total por município entre os anos de 2004 e 2013. Observase que o valor médio dos salários e outras remunerações cresceu, em média, 14,3\% a.a.

\section{TABELA 5 ESTATÍSTICAS DESCRITIVAS DOS SALÁRIOS E OUTRAS REMUNERAÇÕES, VALORES CORRENTES, EM R\$ MIL}

\begin{tabular}{cccccc}
\hline Ano & Média & Mediana & Desvio padrão & Mínimo & Máximo \\
\hline 2004 & $71.641,06$ & $3.342,26$ & $1.101 .459,89$ & - & $67.555 .828,38$ \\
2005 & $81.569,35$ & $4.110,83$ & $1.232 .143,08$ & - & $75.267 .796,59$ \\
2006 & $98.407,89$ & $5.216,56$ & $1.471 .465,41$ & 12,59 & $89.181 .133,84$ \\
2007 & $110.887,61$ & $6.228,25$ & $1.636 .708,62$ & 2,28 & $99.171 .050,06$ \\
2008 & $128.854,13$ & $7.348,09$ & $1.893 .471,47$ & 16,29 & $114.669 .100,24$ \\
2009 & $143.927,64$ & $8.783,80$ & $2.077 .020,31$ & - & $124.377 .680,04$ \\
2010 & $167.492,48$ & $10.260,29$ & $2.376 .854,55$ & 47,70 & $142.150 .179,34$ \\
2011 & $191.989,01$ & $12.049,54$ & $2.702 .458,72$ & 96,66 & $161.769 .713,10$ \\
2012 & $218.414,95$ & $13.972,78$ & $3.054 .433,77$ & 8,03 & $182.963 .919,90$ \\
2013 & $238.091,26$ & $16.038,26$ & $3.350 .990,32$ & 29,50 & $201.301 .606,08$ \\
\hline
\end{tabular}

Fonte: Elaborada pelo autor. 
A Tabela 6 traz as estatísticas descritivas referentes à variável pessoal ocupado assalariado total por município entre os anos de 2004 e 2013. Observa-se que o valor médio do pessoal ocupado assalariado cresceu, em média, $4,9 \%$ a.a.

\section{TABELA 6 ESTATÍSTICAS DESCRITIVAS DA VARIÁVEL PESSOAL OCUPADO ASSALARIADO}

\begin{tabular}{|c|c|c|c|c|c|}
\hline Ano & Média & Mediana & Desvio padrão & Mínimo & Máximo \\
\hline 2004 & 5.608 & 582 & 61.241 & - & 3.543 .743 \\
\hline 2005 & 5.956 & 630 & 64.552 & - & 3.731 .795 \\
\hline 2006 & 6.301 & 683 & 68.326 & 2 & 3.975 .545 \\
\hline 2007 & 6.776 & 732 & 73.570 & 4 & 4.277 .939 \\
\hline 2008 & 7.103 & 751 & 77.457 & 1 & 4.528 .310 \\
\hline 2009 & 7.442 & 830 & 79.913 & - & 4.654 .681 \\
\hline 2010 & 7.961 & 875 & 84.378 & 3 & 4.906 .877 \\
\hline 2011 & 8.368 & 921 & 88.157 & 6 & 5.098 .791 \\
\hline 2012 & 8.568 & 919 & 90.726 & 1 & 5.239 .502 \\
\hline 2013 & 8.598 & 970 & 89.971 & 3 & 5.281 .006 \\
\hline
\end{tabular}

Fonte: Elaborada pelo autor.

A Tabela 7 traz as estatísticas descritivas referentes a unidades locais do município entre os anos de 2004 e 2013. Observa-se que o valor médio das unidades locais do município decresceu, em média, $0,1 \%$ a.a. no período. Também se observa decréscimo de unidades locais no ano de 2006, com recuperação no ano imediatamente seguinte.

\section{TABELA 7 ESTATÍSTICAS DESCRITIVAS DA VARIÁVEL UNIDADES LOCAIS}

\begin{tabular}{|c|c|c|c|c|c|}
\hline Ano & Média & Mediana & Desvio padrão & Mínimo & Máximo \\
\hline 2004 & 1.068 & 191 & 9.375 & 5 & 571.309 \\
\hline 2005 & 1.125 & 203 & 9.845 & 4 & 600.936 \\
\hline 2006 & 857 & 154 & 7.689 & 2 & 472.143 \\
\hline 2007 & 883 & 160 & 7.992 & 6 & 491.553 \\
\hline 2008 & 922 & 166 & 8.416 & 4 & 520.533 \\
\hline 2009 & 971 & 180 & 8.798 & 7 & 544.983 \\
\hline 2010 & 1.029 & 192 & 9.320 & 7 & 577.190 \\
\hline 2011 & 1.032 & 187 & 9.423 & 4 & 584.225 \\
\hline 2012 & 1.049 & 187 & 9.679 & 6 & 599.434 \\
\hline 2013 & 1.054 & 195 & 9.718 & 7 & 618.713 \\
\hline
\end{tabular}

Fonte: Elaborada pelo autor. 
Os dados obtidos podem não apresentar, quando analisados descritivamente, distribuição normal, expondo obliquidade em um dos lados quando da apresentação da distribuição de frequências. Essa ocorrência sugere algum tipo de transformação e a transformação aqui utilizada é a do logaritmo neperiano.

Também, para melhor qualidade de comparação dos valores monetários correntes, utilizam-se seus valores constantes. Assim, recorre-se ao deflator implícito do PIB obtido no IBGE, na Diretoria de Pesquisas da Coordenação de Contas Nacionais e ao Índice Nacional de Preços ao Consumidor (INPC) para os demais valores monetários, também obtido no IBGE, conforme a Tabela 8.

\section{TABELA 8 DEFLATOR IMPLÍCITO DO PIB E INPC ENTRE OS ANOS 2004 A 2013}

\begin{tabular}{|c|c|c|c|c|}
\hline \multirow{2}{*}{ Ano } & \multicolumn{2}{|c|}{ Deflator implícito do PIB } & \multicolumn{2}{|c|}{ INPC } \\
\hline & Percentual & Divisor acumulado & Percentual & Divisor acumulado \\
\hline 2004 & & 1,000 & & 1,000 \\
\hline 2005 & 0,0749 & 1,075 & 0,051 & 1,051 \\
\hline 2006 & 0,0669 & 1,147 & 0,028 & 1,080 \\
\hline 2007 & 0,0640 & 1,220 & 0,052 & 1,136 \\
\hline 2008 & 0,0887 & 1,328 & 0,065 & 1,209 \\
\hline 2009 & 0,0735 & 1,426 & 0,041 & 1,259 \\
\hline 2010 & 0,0857 & 1,548 & 0,065 & 1,341 \\
\hline 2011 & 0,0831 & 1,677 & 0,061 & 1,422 \\
\hline 2012 & 0,0587 & 1,775 & 0,062 & 1,510 \\
\hline 2013 & 0,0651 & 1,891 & 0,056 & 1,594 \\
\hline
\end{tabular}

Fonte: Elaborada pelo autor.

\section{RESULTADOS}

Esta seção apresenta, mediante os três modelos, primeiras diferenças, efeitos fixos e Arellano-Bond, os resultados nas 2 hipóteses testadas.

\subsection{Mínimos quadrados ordinários}

O uso do método de estimação dos MQO das variáveis na primeira diferença (ano a ano) constitui uma ferramenta simples para tratar o problema do viés gerado pela omissão de variáveis, uma vez que, tomando como premissa que a heterogeneidade é constante no tempo, ela será retirada na 
subtração. Uma vantagem desse modelo é que ele remove a heterogeneidade, independente do modelo, de efeitos fixos ou randômicos, para que seja o mais apropriado. Tal método faz com que os coeficientes resultantes do MQO sejam consistentes e não enviesados. Outrossim, os $\Delta u_{i, t}{ }^{4}$ podem ser correlacionados no tempo, indicando que modelos mais avançados devam ser utilizados (Grene, 2008).

- Hipótese H1: O benefício do PBF está associado positivamente com a quantidade de pessoal ocupado assalariado do município.

Modelo 1:

$$
\begin{gathered}
\ln \Delta \text { pop assalariada }= \\
=\beta_{\mathrm{o}}+\beta_{1} \ln \Delta \text { unidades locais }+\beta_{2} \ln \Delta \mathrm{PIB}+\beta_{3} \ln \Delta \text { PBF valor repassado }+\mathrm{u}_{\mathrm{i}}
\end{gathered}
$$

Modelo 2:

$$
\begin{gathered}
\ln \Delta \text { pop assalariada }= \\
=\beta_{\mathrm{o}}+\beta_{1} \ln \Delta \text { unidades locais }+\beta_{2} \ln \Delta \mathrm{PIB}+\beta_{3} \ln \Delta \text { PBF beneficiários }+\mathrm{u}_{\mathrm{i}}
\end{gathered}
$$

A Tabela 9 apresenta os resultados do impacto do PBF no pessoal assalariado do município em

\begin{tabular}{|c|c|c|}
\hline & Modelo 1 & Modelo 2 \\
\hline Ln $\Delta$ unidades locais & $0,4259^{\star \star \star}$ & $0,3929^{\star \star \star}$ \\
\hline Ln $\Delta$ PIB do município & $0,2568^{\star \star \star}$ & $0,2947^{\star \star \star}$ \\
\hline Ln $\triangle$ PBF valores repassados & $0,22149^{\star \star \star}$ & \\
\hline Ln $\triangle$ PBF número de beneficiários & & $0,2033^{\star \star \star}$ \\
\hline Constante & $-3,1462^{\star \star \star}$ & $-1,9495^{\star \star \star}$ \\
\hline $\mathrm{R}^{2}$ & 0,47 & 0,48 \\
\hline
\end{tabular}
valores constantes de 2004.

\section{TABELA 9 IMPACTO DO PBF NO PESSOAL ASSALARIADO DO MUNICÍPIO, LOGARITMO NEPERIANO DAS VARIÁVEIS EM PRIMEIRA DIFERENÇA, VALORES CONSTANTES DE 2004}

Fonte: Elaborada pelo autor.

O modelo 1 indica que, considerando controles como número de unidades locais municipais e o PIB do município, o valor total repassado do PBF está positivamente associado ao número de pessoas ocupadas e assalariadas no município. O aumento de $1 \%$ na diferença do valor repassado entre um ano e outro leva a um acréscimo de $0,22 \%$ na diferença do pessoal assalariado no município entre 2 anos.

${ }^{4} \Delta u_{i, t}$ significa primeira diferença também aplicada ao erro aleatório. 
O modelo 2 mostra que um aumento de $1 \%$ na diferença dos beneficiários entre 2 anos leva a um acréscimo de $0,20 \%$ na diferença do pessoal ocupado entre 2 anos. Os 2 modelos confirmam a hipótese.

- Hipótese H2: O benefício do PBF está associado positivamente com o total dos salários e outras remunerações no município.

Modelo 1:

$\ln \Delta$ salários totais $=$

$=\beta_{\mathrm{o}}+\beta_{1} \ln \Delta$ pop assalariada $+\beta_{2} \ln \Delta \mathrm{PIB}+\beta_{3} \ln \Delta \mathrm{PBF}$ valor repassado $+\mathrm{u}$

Modelo 2:

$\ln \Delta$ salários totais $=$

$=\beta_{\mathrm{o}}+\beta_{1} \ln \Delta$ pop assalariada $+\beta_{2} \ln \Delta \mathrm{PIB}+\beta_{3} \ln \Delta \mathrm{PBF}$ beneficiários $+\mathrm{u}_{\mathrm{i}}$

A Tabela 10 apresenta os resultados do impacto do PBF nos salários totais municipais em valores constantes de 2004.

\section{TABELA 10 IMPACTO DO PBF NOS SALÁRIOS TOTAIS MUNICIPAIS, LOGARITMO NEPERIANO DAS VARIÁVEIS EM PRIMEIRA DIFERENÇA, VALORES CONSTANTES DE 2004}

$\begin{array}{lcc} & \text { Modelo } 1 & \text { Modelo } 2 \\ \text { Ln } \Delta \text { população assalariada } & 0,6614^{\star \star \star} & 0,6332^{\star \star *} \\ \text { Ln } \Delta \text { PIB do município } & 0,2009^{\star \star *} & 0,2279^{\star \star *} \\ \text { Ln } \Delta \text { PBF valores repassados } & 0,1098^{\star \star \star} & \\ \text { Ln } \Delta \text { PBF número de beneficiários } & & 0,1201^{\star \star *} \\ \text { Constante } & 6,6526^{\star \star *} & 7,1032^{\star \star *} \\ \mathrm{R}^{2} & 0,69 & 0,70\end{array}$

Fonte: Elaborada pelo autor.

O modelo 1 indica que o aumento de $1 \%$ na diferença entre 2 anos do valor repassado do PBF leva a um aumento médio de $0,11 \%$ na diferença dos salários totais do município. O modelo 2 indica que um aumento de $1 \%$ na diferença entre 2 anos do número de beneficiários do PBF leva a um aumento médio de $0,12 \%$ na diferença dos salários totais do município. Os 2 modelos confirmam a hipótese.

\subsection{Modelos de dados em painel}

A segunda parte da análise estatística apresenta os resultados do modelo de dados em painel, conjuntos de dados nos quais as mesmas unidades de corte transversal são acompanhadas ao longo do tempo. O teste de Hausman (1978), para as 2 hipóteses, indicam a utilização modelo de efeitos fixos em detrimento do modelo de efeitos randômicos. 
- Hipótese H1: O benefício do PBF está associado positivamente com a quantidade de pessoal ocupado assalariado do município.

Modelo 1:

pop assalariada $=\beta_{\mathrm{o}}+\beta_{1}$ unidades locais $+\beta_{2} \mathrm{PIB}+\beta_{3} \mathrm{PBF}$ beneficiários $+\mathrm{u}_{\mathrm{i}}$

Modelo 2:

pop assalariada $=\beta_{\mathrm{o}}+\beta_{1}$ unidades locais $+\beta_{2} \mathrm{PIB}+\beta_{3}$ PBF valor repassado $+\mathrm{u}_{\mathrm{i}}$

A Tabela 11 apresenta os resultados do impacto do PBF no pessoal ocupado assalariado municipal, em valores constantes de 2004 .

\section{TABELA 11 IMPACTO DO PBF NO PESSOAL OCUPADO ASSALARIADO MUNICIPAL, VALORES CONSTANTES DE 2004}

$\begin{array}{lcc}\text { Variáveis Independentes } & \text { Modelo } 1 & \text { Modelo } 2 \\ \text { Unidades locais } & 4,91^{\star * \star} & 4,49^{* * *} \\ \text { PIB do município } & 0,000000423 & 0,000000371 \\ \text { PBF beneficiários } & 2,97^{\star \star *} & \\ \text { PBF valor repassado } & & 0,00272^{* \star *} \\ \text { Constante } & -3.906,25 & -2.027,73^{\star *}\end{array}$

Fonte: Elaborada pelo autor.

O modelo 1 indica que, considerando controles como PIB municipal e o número de unidades locais do município, um beneficiário adicional do PBF gera um aumento de 2,97 pessoas ocupadas assalariadas no município, com significância de 99\%. O modelo 2 indica que um acréscimo de uma unidade monetária concedida pelo programa acarreta aumento de 0,003 pessoas assalariadas no município, com significância de 99\%. Assim, ambos os modelos confirmam a hipótese de que o PBF está positivamente associado com o número de trabalhadores assalariados no setor formal.

Teste de Hausman. H0: ausência de correlação entre os regressores e o termo de erro.

\section{TABELA 12 TESTE DE HAUSMAN. HIPÓTESE 1, MODEL0 1}

\begin{tabular}{lcccc} 
& EF & ER & EF-ER & SE \\
\hline Unidades locais & 4,917 & 6,715 & $-1,798$ & 0,0319 \\
PBF beneficiários & 2,972 & 2,663 & 0,309 & 0,0113 \\
PIB do município & 0,000000423 & 0,000000566 & $-0,000000143$ \\
Quil $^{2}$ & 4548,06 & & \\
Prob>qui & 0 & & \\
\hline
\end{tabular}

Fonte: Elaborada pelo autor. 


\section{TABELA 13 TESTE DE HAUSMAN. HIPÓTESE 1, MODEL0 2}

\begin{tabular}{lcccr} 
& EF & ER & EF-ER & SE \\
\hline Unidades locais & 4,494 & 6,977 & $-2,483$ & 0,0331 \\
\hline PBF valor repassado & 0,0027 & 0,0026 & 0,0001 & 0,00000464 \\
PIB do município & 0,000000371 & 0,000000501 & $-0,00000013$ \\
Quil $^{2}$ & 6629,41 & & \\
Prob>qui & 0 & & \\
\hline
\end{tabular}

Fonte: Elaborada pelo autor

A Tabela 12 apresenta o teste de Hausman da Hipótese 1 para o modelo 1, onde EF significa efeitos fixos, ER significa efeitos randômicos, EF-ER é a diferença entre ambos e SE é o standard error (erro padrão) e a Tabela 13 apresenta o teste de Hausman da Hipótese 1 para o modelo 2. Em ambos os modelos, o teste de Hausman indica que a hipótese nula deve ser rejeitada (Prob $>$ qui ${ }^{2}<0,05$ ), pois há correlação entre os regressores e o erro. Portanto, deve-se usar o modelo de efeitos fixos.

- Hipótese H2: O benefício do PBF está associado positivamente com o total dos salários e outras remunerações no município.

Modelo 1:

salários totais $=\beta_{\mathrm{o}}+\beta_{1}$ pop assalariada $+\beta_{2} \mathrm{PIB}+\beta_{3} \mathrm{PBF}$ beneficiários $+\mathrm{u}_{\mathrm{i}}$

Modelo 2:

salários totais $=\beta_{\mathrm{o}}+\beta_{1}$ pop assalariada $+\beta_{2} \mathrm{PIB}+\beta_{3} \mathrm{PBF}$ valor repassado $+\mathrm{u}_{\mathrm{i}}$

A Tabela 14 apresenta os resultados do impacto do PBF nos salários totais municipais, em valores constantes de 2004.

\section{TABELA 14 IMPACTO DO PBF NOS SALÁRIOS TOTAIS MUNICIPAIS, VALORES CONSTANTES DE 2004}

\begin{tabular}{ccc} 
Variáveis independentes & Modelo 1 & Modelo 2 \\
\hline População assalariada & $26.076,13^{\star \star \star}$ & $26.028,78^{\star \star \star}$ \\
PIB do município & $0,0115^{\star \star}$ & $0,0114^{\star \star *}$ \\
PBF beneficiários & $16.448,24 \wedge$ & 12,5 \\
PBF valor repassado & & $-1.046 .000 .000^{\star * \star}$ \\
Constante & $-1.040 .000 .000^{* * *}$ &
\end{tabular}

Fonte: Elaborada pelo autor. 
O modelo 1 indica que, controlando pelo PIB municipal e o número de pessoas assalariadas no setor formal, o aumento de um beneficiário do PBF leva a um acréscimo de aproximadamente R\$ 16.500,00 nos salários totais dos municípios. No entanto, o coeficiente da equação possui segurança inferior ao geralmente aceito em trabalhos de Ciências Sociais. O modelo 2 aponta uma relação positiva entre o valor repassado do PBF e os salários totais do município. No entanto, o coeficiente não é estatisticamente diferente de zero. Assim, os modelos acima demonstram relação positiva entre o PBF e os salários totais, porém, não é possível aceitar de maneira cabal a Hipótese 2 com base nas estimativas acima.

Teste de Hausman. H0: ausência de correlação entre os regressores e o termo de erro.

\section{TABELA 15 TESTE DE HAUSMAN. HIPÓTESE 2, MODEL0 1}

\begin{tabular}{lcccc} 
& EF & ER & EF-ER & SE \\
\hline População assalariada & 26028,78 & 20884,44 & $-1,798$ & 0,0319 \\
PBF valor repassado & 0,0114 & 0,01274 & 0,309 & 0,0113 \\
PIB do município & 12,5502 & 16,2723 & $-0,000000143$ \\
Quiं & 17326,12 & & \\
Prob>qui & 0 & & \\
\hline
\end{tabular}

Fonte: Elaborada pelo autor.

\section{TABELA 16 TESTE DE HAUSMAN. HIPÓTESE 2, MODEL0 2}

\begin{tabular}{lcccc} 
& EF & ER & EF-ER & SE \\
\hline População assalariada & 26028,78 & 20884,44 & $-1,798$ & 0,0319 \\
PBF valor repassado & 0,0114 & 0,01274 & 0,309 & 0,0113 \\
PIB do município & 12,5502 & 16,2723 & $-0,000000143$ \\
Quil $^{2}$ & 17326,12 & & \\
Prob>quil & 0 & & \\
\hline
\end{tabular}

Fonte: Elaborada pelo autor.

A Tabela 15 apresenta o teste de Hausman da Hipótese 2 para o modelo 1 e a Tabela 16 apresenta o teste de Hausman da Hipótese 2 para o modelo 2. Em ambos os modelos, o teste de Hausman indica que a hipótese nula deve ser rejeitada (Prob $>$ qui $\left.^{2}<0,05\right)$, pois há correlação entre os regressores e o erro. Portanto, deve-se usar o modelo de efeitos fixos. 


\subsection{Modelos dinâmicos de Arellano-Bond}

As mesmas duas hipóteses do estudo são testadas com modelos dinâmicos, que incluem a variável dependente defasada como regressor e as variáveis dependentes defasadas em alguns períodos. A decisão de incluir a variável dependente defasada pode basear-se em argumentos teóricos pois, se há razões para supor que os valores correntes das variáveis dependentes são altamente dependentes dos valores passados, a inclusão dessa defasagem reduz o problema de viés devido a omissão de variáveis. Em modelos dinâmicos dessa natureza, não se deve utilizar os modelos de efeito fixos ou randômicos, pois, por construção, a variável dependente defasada é correlacionada com o erro. Essa presença de endogeneidade, faz com que os estimadores sejam inconsistentes. Nesse caso, o método de estimação Arellano-Bond é o mais indicado. Esse estimador utiliza a o método de momentos generalizados (MMG) e fornece estimadores eficientes em um modelo dinâmico.

- Hipótese H1: O benefício do PBF está associado positivamente com a quantidade de pessoal ocupado assalariado do município.

Modelo 1:

pop assalariada $=\beta_{\mathrm{o}}+\beta_{1}$ pop assalariada ${ }_{\mathrm{t}-1}+\beta_{2}$ unidades locais $_{\mathrm{t}}+\beta_{3}$ unidades locais $_{\mathrm{t}-1}+$ $\beta_{4}$ unidades locais ${ }_{\mathrm{t}-2}+\beta_{5}$ PIB $_{\mathrm{t}}+\beta_{6} \mathrm{PIB}_{\mathrm{t}-1}+\beta_{7}$ PIB $_{\mathrm{t}-2}+\beta_{8}$ beneficiários $_{\mathrm{t}}+$ $\beta_{9}$ beneficiários $_{\mathrm{t}-1}+\beta_{10}$ beneficiários $_{\mathrm{t}-2}+\mathrm{u}_{\mathrm{i}}$

Modelo 2:

pop assalariada $=\beta_{\mathrm{o}}+\beta_{1}$ pop assalariada $\mathrm{t}_{\mathrm{t}-1}+\beta_{2}$ unidades locais ${ }_{\mathrm{t}}+\beta_{3}$ unidades locais ${ }_{\mathrm{t}-1}$ $+\beta_{4}$ unidades locais $_{\mathrm{t}-2}+\beta_{5}$ PIB $_{\mathrm{t}}+\beta_{6} \mathrm{PIB}_{\mathrm{t}-1}+\beta_{7} \mathrm{PIB}_{\mathrm{t}-2}+\beta_{8}$ valor repassado $_{\mathrm{t}}+\beta_{9}$ valor repassado $_{\mathrm{t}-1}+\beta_{10}$ valor repassado $_{\mathrm{t}-2}+\mathrm{u}_{\mathrm{i}}$

A Tabela 17 apresenta 2 modelos dinâmicos para o teste da Hipótese 1 deste estudo.

\section{TABELA 17 IMPACTO DO PBF NO PESSOAL OCUPADO ASSALARIADO MUNICIPAL, VALORES CONSTANTES DE 2004, POR MODELO DINÂMICO DE ARELLANO-BOND}

$\begin{array}{lcc}\text { População assalariada } & \text { Modelo } 1 & \text { Modelo } 2 \\ \text { L1 } & 0,21^{* \star \star} & 0,17^{\star \star \star} \\ \text { Unidades locais } & 7,85^{\star \star \star} & 7,79^{\star \star \star} \\ \text { L1 } & 0,27 & -0,49 \\ \text { L2 } & 0,24 & -0,049 \\ \text { PIB do município } & -0,000000141 & -0,000000119 \\ \text { L1 } & -0,00000000959 & 0,0000000217 \\ \text { L2 } & 0,000000145^{*} & 0,000000166^{*} \\ \text { PBF beneficiários } & 0,29 & \\ \text { L1 } & 0,19 & \\ \text { L2 } & 0,56^{\star \star \star} & 0,0003^{* \star *} \\ \text { PBF valor repassado } & & 0,0002 \\ \text { L1 } & & 0,0006^{*}\end{array}$

Fonte: Elaborada pelo autor. 
A abreviação L1 indica que a variável foi defasada em um período, enquanto L2 indica que a variável foi defasada em 2 períodos. Os modelos mostram que há um efeito defasado na resposta: em ambos os modelos, os coeficientes das variáveis beneficiários do PBF e valores totais repassados do PBF são significativos e possuem maior magnitude quando estão defasados em 2 anos.

Teste de robustez. H0: Não há autocorrelação dos erros em primeira diferença.

\section{TABELA 18 TESTE DE AUTOCORRELAÇÃO DOS ERROS EM PRIMEIRA DIFERENÇA DA HIPÓTESE 1}

\begin{tabular}{ccc} 
& Modelo 1 & Modelo 2 \\
\hline Prob $>$ z & 0,024 & 0,0203
\end{tabular}

Fonte: Elaborada pelo autor.

A Tabela 18 apresenta o teste de autocorrelação dos erros em primeira diferença. Deve-se rejeitar H0. Assim, há evidências de que as premissas do modelo Arellano-Bond são respeitadas.

- Hipótese H2: O benefício do PBF está associado positivamente com o total dos salários e outras remunerações no município.

Modelo 1:

salários totais $=\beta_{\mathrm{o}}+\beta_{1}$ salários totais ${ }_{\mathrm{t}-1}+\beta_{2}$ unidades locais $_{\mathrm{t}}+\beta_{3}$ unidades locais $_{\mathrm{t}-1}+$

$\beta_{4}$ unidades locais ${ }_{\mathrm{t}-2}+\beta_{5}$ PIB $_{\mathrm{t}}+\beta_{6} \mathrm{PIB}_{\mathrm{t}-1}+\beta_{7} \mathrm{PIB}_{\mathrm{t}-2}+\beta_{8}$ beneficiários $_{\mathrm{t}}+\beta_{9}$ beneficiários $\mathrm{t}-1+\beta_{10}$ beneficiários $_{\mathrm{t}-2}+\mathrm{u}_{\mathrm{i}}$

Modelo 2:

salários totais $=\beta_{\mathrm{o}}+\beta_{1}$ salários totais ${ }_{\mathrm{t}-1}+\beta_{2}$ unidades locais $_{\mathrm{t}}+\beta_{3}$ unidades locais $_{\mathrm{t}-1}+$ $\beta_{4}$ unidades locais ${ }_{\mathrm{t}-2}+\beta_{5}$ PIB $_{\mathrm{t}}+\beta_{6}$ PIB $_{\mathrm{t}-1}+\beta_{7}$ PIB $_{\mathrm{t}-2}+\beta_{8}$ valor repassado $_{\mathrm{t}}+\beta_{9}$ valor repassado $_{\mathrm{t}-1}+\beta_{10}$ valor repassado $_{\mathrm{t}-2}+\mathrm{u}_{\mathrm{i}}$

A Tabela 19 apresenta os resultados do impacto do PBF nos salários totais municipais, em valores constantes de 2004.

\section{TABELA 19 IMPACTO DO PBF NOS SALÁRIOS TOTAIS MUNICIPAIS, VALORES CONSTANTES DE 2004 POR MODELO DINÂMICO DE ARELLANO-BOND}

$\begin{array}{lcc} & \text { Modelo } 1 & \text { Modelo } 2 \\ \text { Salários totais } & - & - \\ \text { L1 } & 0,57^{\star \star \star} & 0,55^{\star \star \star} \\ \text { Unidades locais } & 116.956,4^{\star \star \star} & 116.757,3^{\star \star \star}\end{array}$




$\begin{array}{lcc} & \text { Modelo } 1 & \text { Modelo } 2 \\ \text { L1 } & 29.356,79^{\star \star \star} & 29.450,9^{\star \star \star} \\ \text { L2 } & 2.807,45^{\star \star \star} & -4.693,75 \\ \text { PIB do município } & & 0,0012 \\ \text { L1 } & 0,0016 & 0,0008 \\ \text { L2 } & 0,004^{\star \star} & 0,0048^{\star \star} \\ \text { PBF beneficiários } & 7.342,5^{\star \star \star} & \\ \text { L1 } & 7.137,21^{\star \star} & \\ \text { L2 } & 11.790,01^{\star \star \star} & \\ \text { PBF valor repassado } & & 14,43^{\star \star \star} \\ \text { L1 } & & -1,19 \\ \text { L2 } & & -4,78\end{array}$

Fonte: Elaborada pelo autor.

O modelo 1 também indica o caráter dinâmico do impacto do número de beneficiados no PBF e os salários formais totais do município. O coeficiente da variável explicativa defasada em dois anos indica que o acréscimo de um beneficiário do PBF leva, em média, a um aumento de R \$11.790,01 no salário total do município. Os coeficientes se mantêm positivos e significantes para a variável em nível. Para a variável defasada em um ano, no entanto, o impacto se reduz para R\$7.137,21. No modelo 2, o efeito dinâmico não aparece. Apenas o coeficiente da variável em nível é significativa e indica que um aumento de $\mathrm{R} \$ 1,00$ no valor total repassado do PBF leva a um aumento de $\mathrm{R}$ \$ 14,00 nos salários formais totais dos municípios. Por fim, o teste de robustez também indica que há evidências de que as premissas do modelo Arellano-Bond são respeitadas.

Teste de robustez. H0: Não há autocorrelação dos erros em primeira diferença.

\section{TABELA 20 TESTE DE AUTOCORRELAÇÃO DOS ERROS EM PRIMEIRA DIFERENÇA DA HIPÓTESE 2}

\begin{tabular}{ccc} 
& Modelo 1 & Modelo 2 \\
\hline Prob $>$ z & 0,0084 & 0,0053
\end{tabular}

Fonte: Elaborada pelo autor.

A Tabela 20 apresenta os resultados do teste de autocorrelação dos erros em primeira diferença. Deve-se rejeitar H0. Assim, há evidências de que as premissas do modelo Arellano-Bond são respeitadas. 


\section{CONSIDERAÇÕES FINAIS}

Conforme os resultados obtidos, os 3 modelos utilizados (primeiras diferenças; efeitos fixos; e ArellanoBond) indicam a associação positiva dos benefícios do PBF do município com o pessoal ocupado assalariado e com os salários e outros rendimentos no município.

Do ponto de vista acadêmico, entende-se como contribuição que, ao usar os dados específicos totais de cada um dos municípios da União ao invés de ter por base os dados da PNAD ou do Censo do IBGE, esta pesquisa possibilita a confrontação com conclusões de estudos anteriores, desenvolvidos mediante o uso de bases de dados alternativas. Nesse sentido, o resultado ora apresentado corrobora resultados anteriores, obtidos com bases alternativas, que indicam a associação positiva do PBF com o mercado de trabalho.

Uma segunda contribuição acadêmica é a aplicação dos 3 modelos econométricos, logaritmos neperianos das primeiras diferenças, modelo de dados em painel de efeitos fixos e modelo de dados em painel dinâmico de Arellano-Bond, cuja convergência dos resultados solidifica a pesquisa.

Do ponto de vista de gestão de política pública, entende-se como real e efetiva contribuição a constatação de que o PBF está positivamente associado ao mercado de trabalho formal dos municípios brasileiros, tanto no que se refere ao aumento do pessoal ocupado assalariado como mediante o aumento dos salários e outros rendimentos. Tais resultados reforçam estudos anteriores que sugerem que as transferências de renda provenientes do PBF podem causar um aumento de salários em um novo equilíbrio de mercado do município. Acrescenta-se à contribuição anterior a constatação que os resultados obtidos indicam que o benefício do PBF não causa efeitos adversos em relação às decisões relacionadas à oferta de trabalho.

Assim, preserva-se a expectativa de manutenção desse programa de transferência de rendas com condicionalidades, tanto como elemento gerador de benefícios sociais, caracterizados no combate à pobreza absoluta, melhoria da educação, da saúde e no combate à fome das famílias, quanto como fator endógeno contribuinte do crescimento do pessoal ocupado assalariado e com os salários e outros rendimentos no município.

Algumas limitações desta pesquisa devem ser mencionadas. Uma primeira limitação diz respeito à não consideração da associação do trabalhador de um município com seu endereço, uma vez que os dados relativos ao indivíduo podem estar alocados, quanto ao benefício do PBF, em um município e, como pessoa ocupada formalmente, em município distinto. Uma segunda limitação é a da pesquisa versar apenas sobre o trabalho formal, uma vez que a fonte utilizada oferece apenas esse dado. Assim, o efeito substituição, oriundo do trabalho informal, não é considerado.

Por fim, algumas sugestões podem ser oferecidas para a continuidade deste estudo: a) pesquisas utilizando dados de 2014 e 2016, que incorporam uma nova tendência da taxa de desemprego; b) pesquisas englobando a análise individual dos municípios, buscando observar em quais ambientes municipais há uma maior aderência aos resultados ora apresentados; e c) pesquisa de possíveis características no conjunto de municípios onde a associação positiva é mais latente. 


\section{REFERÊNCIAS}

Alzúa, M. L., Cruces, G., \& Ripani, L. (2012). Welfare programs and labor supply in developing countries: experimental evidence from Latin America. Journal of Population Economics, 26(4), 1255-1284.

Arellano, M., \& Bond, S. (1991). Some tests of specification for panel data: Monte Carlo evidence and an application to employment equations. Review of Economic Studies, 58, 277-297.

Arretche, M. (1995). Emergência e desenvolvimento do welfare state: teorias explicativas. Revista Brasileira de Informação Bibliográfica em Ciências Sociais, 39, 3-40.

Asfaw, S., Davis, B., Dewbre, J., Handa, S., \& Winters, P. (2014). Cash transfer programme, productive activities and labour supply: evidence from randomized experiment in Kenya. Journal of Development Studies, 50(8), 1172-1196.

Barbosa, A. L. N. H., \& Corseuil, C. H. L. (2014, abril). Bolsa Família, escolha ocupacional e informalidade no Brasil (Texto para Discussão IPEA n. 1948). Recuperado de http://repositorio.ipea.gov.br/ bitstream/11058/3026/1/TD_1948.pdf

Barro, R. J. (1990). Government spending in a simple model of endogeneous growth. Journal of Political Economy, 98 (S5).

Barro, R. J. (1991, maio). Economic Growth in a Cross Section of Countries. The Quarterly Journal of Economics, 106(2), 407-443.

Brito, A., \& Kerstenetzky, C. L. (2010). Programa Bolsa Família e mercado de trabalho: considerações metodológicas e substantivas. In Anais do 380 Encontro Nacional de Economia, Salvador, BA.

Bussmann, T. B., \& Risco, G. R. M. (2012). Mensuração da desigualdade e do crescimento no estado de Minas Gerais: estimação da curva de Kuznets. In Anais do 150 Seminário sobre a Economia Mineira, Diamantina, MG.

Cavalcanti, D. M., Costa, E. M., Silva, J. L. M., \& Sampaio, R. M. B. (2016). Impactos do Programa Bolsa Família na renda e na oferta de trabalho das famílias pobres: uma abordagem usando o efeito quantílico de tratamento. Economia Aplicada, 20(2), 173-201.

Cavalcanti, T., \& Corrêa, M. (2010). Cash transfers and the labor market. Revista Brasileira de Economia, 64(2), 175-190.
Constituição da República Federativa do Brasil, de 5 de outubro de 1988. (1988). Brasília, DF.

Denes, G., Komatsu, B. K., \& Menezes, N., Filho. (2016, agosto). Uma avaliação dos impactos macroeconômicos e sociais de programas de transferência de renda nos municípios brasileiros (Policy Paper Insper n. 21). Recuperado de https:// www.insper.edu.br/wp-content/uploads/2018/09/ Avaliacao-Impactos-Macroeconomicos-SociaisTransferencia-Renda-Municipios-Brasileiros.pdf

Draibe, S. M. (2009). Programas de transferências condicionadas de renda. In F. H. Cardoso, A. Alejandro (Eds.), América Latina: desafios da democracia e do desenvolvimento - políticas sociais para além da crise. Rio de Janeiro, RJ: Campus.

Easterly, W., \& Rebelo, S. (1993). Fiscal policy and economic growth: an empirical investigation. Journal of Monetary Economics, 32, 417-458.

Economic Commission for Latin America and the Caribbean, \& International Labour Organization. (2015, maio). Employment situation in Latin America and the Caribbean (Number 12). Recuperado de https://repositorio.cepal.org/bitstream/ handle/11362/38053/1/S1500310_en.pdf

Fleury, S. (1994). Estado sem cidadãos: seguridade social na América Latina. Rio de Janeiro, RJ: Fundação Oswaldo Cruz.

Foguel, M. N., \& Barros, R. P. (2010). The effects of conditional cash transfer programmes on adult labour supply: an empirical analysis using a time-series-cross-section sample of Brazilian municipalities. Estudos Econômicos, 40(2), 259-293.

Grene, W. H. (2008). Econometric analysis. Upper Saddle River, NJ: Pearson Prentice Hall.

Hausman, J. A. (1978, novembro). Specification Test in Econometrics. Econometrica, 46(6), 1251-1271.

Jacinto, P. A., \& Tejada, C. A. O. (2009). Desigualdade de renda e crescimento econômico nos municípios da região Nordeste do Brasil: o que os dados têm a dizer? Revista Econômica do Nordeste, 40(1), 61-80.

Kabber, N., \& Waddington, H. (2015). Economic impacts of conditional cash transfer programmes: a systematic review and meta-analysis. Journal of Development Effectiveness, 7(3), 290-303.

Kerstenetzky, C. L. (2011). Welfare state e desenvolvimento. Dados: Revista de Ciências Sociais, 54(1), 129-156. 
King, G., Keohane, R. O., \& Verba, S. (1996). Designing social inquiry: scientific inference in qualitative research. Princeton, NJ: Princeton University Press.

Lucas, R. E., Jr. (1988). On the mechanics of economic development. Journal of Monetary Economics, 22, 3-42.

Marinho, E., \& Mendes, S. (2013). The impact of government income transfers on the Brazilian job market. Estudos Econômicos, 43(1), 29-50.

Nunes, J. A., \& Mariano, J. L. (2015). Efeitos dos programas de transferência de renda sobre a oferta de trabalho não agrícola na área rural da região Nordeste. Revista de Economia e Sociologia Rural, 53(1), 71-90.

Okun, A. M. (1975). Equality and efficiency: the big tradeoff. Washington, DC: Brookings Institution Press.

Oliveira, L. F. B., \& Soares, S. S. D. (2012, maio). $O$ que se sabe sobre os efeitos das transferências de renda sobre a oferta de trabalho (Texto para Discussão IPEA n. 1738). Recuperado de http:// www.ipea.gov.br/portal/index.php?option=com content\&view=article\&id $=15785$

Pontusson, J. (2005). Inequality and prosperity: social Europe vs. liberal America. New York, NY: Cornell University Press.
Rands Barros, A., \& Athias, D. (2013). Salário mínimo, Bolsa Família e desempenho relativo recente da economia do Nordeste. Revista de Economia Política, 33(1), 179-199.

Ribas, R., \& Soares, F. (2011, March 9). Is the effect of conditional transfers on labor supply negligible everywhere? Recuperado de http://conference.iza. org/conference_files/worldb2011/ribas_r6802.pdf

Romer, P. M. (1986). Increasing returns and longrun growth. The Journal of Political Economy, 94(5), 1002-1037.

Santos, D. B., Leichsenring, A. R., Menezes, N., Filho, \& Mendes-da-Silva, W. (2017). Os efeitos do Programa Bolsa Família sobre a duração do emprego formal dos indivíduos de baixa renda. Revista de Administração Pública, 51(5), 708-733.

Skoufias, E., \& Di Maro, V. (2008). Conditional cash transfers, adult work incentives, and poverty. The Journal of Development Studies, 44(7): 935-960.

Soares, S., \& Sátyro, N. (2009). O Programa Bolsa Família: desenho institucional, impactos e possibilidades futuras. Brasília, DF: Instituto de Pesquisa Econômica Aplicada.

Tavares, P. A. (2010). Efeito do Programa Bolsa Família sobre a oferta de trabalho das mães. Economia e Sociedade, 19(3), 613-635.

\section{Carlos Barbosa Correa Junior}

https://orcid.org/0000-0002-3614-2153

Doutor em Administração pelo Programa de Estudos Pós-Graduação em Administração da Pontifícia Universidade Católica de São Paulo (PUC-SP). E-mail: carlosbcj5454@gmail.com

\section{Leonardo Nelmi Trevisan}

\section{https://orcid.org/0000-0002-0914-3679}

Doutor em Ciência Política pelo Programa de Estudos Pós-Graduação em Administração da Pontifícia Universidade Católica de São Paulo (PUC-SP). E-mail: Intrevisan@pucsp.br

\section{Cristina Helena Pinto de Mello}

https://orcid.org/0000-0002-2002-0645

Doutora em Economia pelo Programa de Mestrado em Comportamento do Consumidor da Escola Superior de Propaganda e Marketing (ESPM). E-mail: chmellop@gmail.com 